\title{
A Unique Common Coupled Fixed Point Theorem for Four Maps in Partial b-Metric- Like Spaces
}

\author{
Mohammad S. Khan ${ }^{1 *}$, Konduru P.R. Rao ${ }^{2}$ and Kandipalli V.S. Parvathi ${ }^{3}$
}

${ }^{1}$ Department of Mathematics and Statistics, College of Science, Sultan Qaboos University, P.O. Box 36, PC 123, Al-Khod, Muscat, Sultanate of Oman. ${ }^{2}$ Department of Mathematics, Acharya Nagarjuna University, Nagarjuna Nagar -522 510, A.P., India. ${ }^{3}$ Department of Applied Mathematics,Krishna University-M.R.Appa Row P.G.Center, Nuzvid-521 201, Andhra Pradesh, India. *Email: mohammad@squ.edu.om.

\begin{abstract}
We prove the existence of a unique common coupled fixed point theorem for four mappings satisfying a general contractive condition on partial b-metric-like spaces. We also give an example to illustrate our main theorem. Our theorem generalizes and improves the theorem of [1].
\end{abstract}

Keywords: b-Metric-like space; Coupled fixed point; w-Compatible maps.

نظرية النقطة الثابتة المزدوجة العامة لاربعة اقترانات في شبه الفراغ الجزئي ب

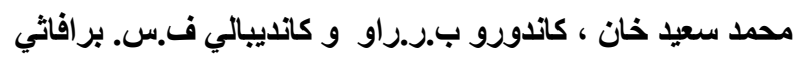

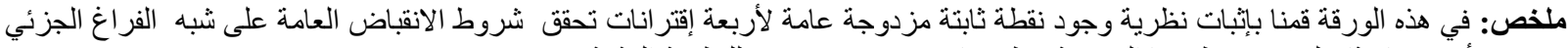

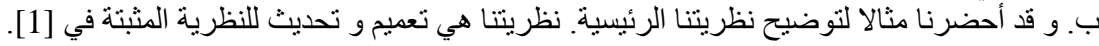

\section{Introduction and Preliminaries}

كلمات مفتاحية: شبه الفراغ الجزئي ب ، نقطة ثابتة مزدوجةو الدوال المتو افقة توافق W.

$\mathbf{T}$ he concept of b-metric space was introduced by Czerwik [2] as follows:

Definition 1.1 [2]: A b-metric on a non-empty set $X$ is a function $d: X \times X \rightarrow[0, \infty)$ such that for all $x, y, z \varepsilon X$ and a constant $\mathrm{k} \geq 1$ the following three conditions hold true:

(i) $d(x, y)=0$ if and only if $x=y$,

(ii) $\mathrm{d}(\mathrm{x}, \mathrm{y})=\mathrm{d}(\mathrm{y}, \mathrm{x})$,

(iii) $\mathrm{d}(\mathrm{x}, \mathrm{y}) \leq \mathrm{k}[\mathrm{d}(\mathrm{x}, \mathrm{z})+\mathrm{d}(\mathrm{z}, \mathrm{y})]$.

The triad $(X, d, k)$ is called a b-metric space.

Alghamdi et al. [3] introduced the concept of b-metric -like spaces and proved some fixed point theorems for a single map.

Definition 1.2 [3]: A b-metric-like on a non-empty set $X$ is a function $d: X \times X \rightarrow[0, \infty)$ such that for all $x, y, z \varepsilon X$ and a constant $\mathrm{k} \geq 1$ the following three conditions hold true:

(i) $d(x, y)=0$ implies $x=y$,

(ii) $\mathrm{d}(\mathrm{x}, \mathrm{y})=\mathrm{d}(\mathrm{y}, \mathrm{x})$,

(iii) $\mathrm{d}(\mathrm{x}, \mathrm{y}) \leq \mathrm{k}[\mathrm{d}(\mathrm{x}, \mathrm{z})+\mathrm{d}(\mathrm{z}, \mathrm{y})]$.

The triad $(\mathrm{X}, \mathrm{d}, \mathrm{k})$ is called a b-metric-like space.

Mathews [4] introduced the concept of a partial metric space as follows:

Definition 1.3 [4]: A mapping $p: X \times X \rightarrow[0, \infty)$, where $X$ is a non-empty set, is said to be a partial metric on $\mathrm{X}$ if for any $\mathrm{x}, \mathrm{y}, \mathrm{z} \in \mathrm{X}$ the following are satisfied:

(i) $\quad x=y$ if and only if $p(x, x)=p(x, y)=p(y, y)$,

(ii) $\mathrm{p}(\mathrm{x}, \mathrm{x}) \leq \mathrm{p}(\mathrm{x}, \mathrm{y})$,

(iii) $\mathrm{p}(\mathrm{x}, \mathrm{y})=\mathrm{p}(\mathrm{y}, \mathrm{x})$, 
(iv) $\mathrm{p}(\mathrm{x}, \mathrm{y}) \leq \mathrm{p}(\mathrm{x}, \mathrm{z})+\mathrm{p}(\mathrm{z}, \mathrm{y})-\mathrm{p}(\mathrm{z}, \mathrm{z})$.

The pair (X, p) is called a partial metric space.

Now we give the following definition by combining the Definitions 1.2 and 1.3.

Definition 1.4: A partial b-metric-like on a non-empty set $X$ is a function $p: X \times X \rightarrow[0, \infty)$ such that for all $x, y, z \varepsilon$ $\mathrm{X}$ and a constant $\mathrm{k} \geq 1$ the following are satisfied:

$\left(\mathrm{p}_{1}\right) \mathrm{p}(\mathrm{x}, \mathrm{y})=0$ implies $\mathrm{x}=\mathrm{y}$,

$\left(\mathrm{p}_{2}\right) \mathrm{p}(\mathrm{x}, \mathrm{x}) \leq \mathrm{p}(\mathrm{x}, \mathrm{y}), \mathrm{p}(\mathrm{y}, \mathrm{y}) \leq \mathrm{p}(\mathrm{x}, \mathrm{y})$,

$\left(\mathrm{p}_{3}\right) \mathrm{p}(\mathrm{x}, \mathrm{y})=\mathrm{p}(\mathrm{y}, \mathrm{x})$

$\left(\mathrm{p}_{4}\right) \mathrm{p}(\mathrm{x}, \mathrm{y}) \leq \mathrm{k}[\mathrm{p}(\mathrm{x}, \mathrm{z})+\mathrm{p}(\mathrm{z}, \mathrm{y})-\mathrm{p}(\mathrm{z}, \mathrm{z})]$.

The triad $(X, p, k)$ is called a partial b-metric-like space.

Definition 1.5: Let $(X, p, k)$ be a partial b-metric-like space and let $\left\{x_{n}\right\}$ be a sequence in $X$ and $x \varepsilon X$. The sequence $\left\{x_{n}\right\}$ is said to be convergent to $x$ if

$\lim _{n \rightarrow \infty} \mathrm{p}\left(\mathrm{x}_{\mathrm{n}}, \mathrm{x}\right)=\mathrm{p}(\mathrm{x}, \mathrm{x})$.

Definition 1.6: Let $(\mathrm{X}, \mathrm{p}, \mathrm{k})$ be a partial b-metric-like space.

(i) A sequence $\left\{x_{n}\right\}$ in $(X, p, k)$ is said to be a Cauchy sequence if $\lim _{n, m \rightarrow \infty} p\left(x_{n}, x_{m}\right)$ exists and is finite.

(ii) A partial b-metric-like space $(X, p, k)$ is said to be complete if every Cauchy sequence $\left\{x_{n}\right\}$ in $X$ converges to a point $\mathrm{x} \& \mathrm{X}$ so that

$\lim _{n, m \rightarrow \infty} \mathrm{p}\left(\mathrm{x}_{\mathrm{n}}, \mathrm{x}_{\mathrm{m}}\right)=\mathrm{p}(\mathrm{x}, \mathrm{x})=\lim _{n \rightarrow \infty} \mathrm{p}\left(\mathrm{x}_{\mathrm{n}}, \mathrm{x}\right)$.

One can prove easily the following remark.

Remark 1.7: Let $(X, p, k)$ be a partial b-metric-like space and $\left\{x_{n}\right\}$ be a sequence in $X$ such that $\lim _{n \rightarrow \infty} p\left(x_{n}, x\right)=0$. Then

(i) $\mathrm{x}$ is unique,

(ii) $\frac{1}{k} \mathrm{p}(\mathrm{x}, \mathrm{y}) \leq \lim _{n \rightarrow \infty} \mathrm{p}\left(\mathrm{x}_{\mathrm{n}}, \mathrm{y}\right) \leq \mathrm{k} \mathrm{p}(\mathrm{x}, \mathrm{y})$ for all $\mathrm{y} \varepsilon \mathrm{X}$,

(iii) $\mathrm{p}\left(\mathrm{x}_{\mathrm{n}}, \mathrm{x}_{0}\right) \leq \mathrm{kp}\left(\mathrm{x}_{0}, \mathrm{x}_{1}\right)+\mathrm{k}^{2} \mathrm{p}\left(\mathrm{x}_{1}, \mathrm{x}_{2}\right)+\cdots+\mathrm{k}^{\mathrm{n}-1} \mathrm{p}\left(\mathrm{x}_{\mathrm{n}-2}, \mathrm{x}_{\mathrm{n}-1}\right)$

$$
+\mathrm{k}^{\mathrm{n}} \mathrm{p}\left(\mathrm{x}_{\mathrm{n}-1}, \mathrm{x}_{\mathrm{n}}\right) \text { whenever }\left\{x_{k}\right\}_{k=0}^{n} \varepsilon \mathrm{X} .
$$

Let $(X, p, k)$ be a partial b-metric-like space and $F, G: X \times X$ and $f, g: X \rightarrow X$. For $x, y, u, v \varepsilon X$, we denote

$$
\mathrm{M}_{\mathrm{u}, \mathrm{v}}^{\mathrm{x}, \mathrm{y}}=\min \left\{\begin{array}{c}
\mathrm{p}(\mathrm{fx}, \mathrm{gu}), \mathrm{p}(\mathrm{fy}, \mathrm{gv}), \mathrm{p}(\mathrm{fx}, \mathrm{F}(\mathrm{x}, \mathrm{y})), \mathrm{p}(\mathrm{fy}, \mathrm{F}(\mathrm{y}, \mathrm{x})), \\
\mathrm{p}(\mathrm{gu}, \mathrm{G}(\mathrm{u}, \mathrm{v})), \mathrm{p}(\mathrm{gv}, \mathrm{G}(\mathrm{v}, \mathrm{u})), \\
\frac{1}{2 \mathrm{k}}[\mathrm{p}(\mathrm{fx}, \mathrm{G}(\mathrm{u}, \mathrm{v}))+\mathrm{p}(\mathrm{gu}, \mathrm{F}(\mathrm{x}, \mathrm{y}))], \\
\frac{1}{2 \mathrm{k}}[\mathrm{p}(\mathrm{fy}, \mathrm{G}(\mathrm{v}, \mathrm{u}))+\mathrm{p}(\mathrm{gv}, \mathrm{F}(\mathrm{y}, \mathrm{x}))]
\end{array}\right\} .
$$

and

$$
m_{u, v}^{x, y}=\max \left\{\begin{array}{c}
\mathrm{p}(\mathrm{fx}, \mathrm{F}(\mathrm{x}, \mathrm{y})), \mathrm{p}(\mathrm{fy}, \mathrm{F}(\mathrm{y}, \mathrm{x})) \\
\mathrm{p}(\mathrm{gu}, \mathrm{G}(\mathrm{u}, \mathrm{v})), \mathrm{p}(\mathrm{gv}, \mathrm{G}(\mathrm{v}, \mathrm{u})) \\
\frac{1}{\mathrm{k}} \mathrm{p}(\mathrm{fx}, \mathrm{G}(\mathrm{u}, \mathrm{v})), \frac{1}{\mathrm{k}} \mathrm{p}(\mathrm{gu}, \mathrm{F}(\mathrm{x}, \mathrm{y})), \\
\frac{1}{\mathrm{k}} \mathrm{p}(\mathrm{fy}, \mathrm{G}(\mathrm{v}, \mathrm{u})), \frac{1}{\mathrm{k}} \mathrm{p}(\mathrm{gv}, \mathrm{F}(\mathrm{y}, \mathrm{x}))
\end{array}\right\}
$$

Recently Bhaskar and Lakshmikantham [5] introduced the concept of coupled fixed point and discussed some problems of the uniqueness of a coupled fixed point and applied their results to the problems of the existence and uniqueness of a solution for the periodic boundary value problems. Later Lakshmikantham and Ciric [6] proved some coupled coincidence and coupled common fixed point results in partially ordered metric spaces. 


\section{A UNIQUE COMMON COUPLED FIXED POINT THEOREM}

Definition 1.8 [6] An element (x, y) $\varepsilon \quad X \times X$ is called

(i) a coupled coincident point of mappings $F: X \times X \rightarrow X$ and $g: X \rightarrow X$ if $g x=F(x, y)$ and $g y=F(y, x)$.

(ii) a common coupled fixed point of mappings $\mathrm{F}: \mathrm{X} \times \mathrm{X} \rightarrow \mathrm{X}$ and $\mathrm{g}: \mathrm{X} \rightarrow \mathrm{X}$

if $x=g x=F(x, y)$ and $y=g y=F(y, x)$.

Definition 1.9 [7] The mappings $\mathrm{F}: \mathrm{X} \times \mathrm{X} \rightarrow \mathrm{X}$ and $\mathrm{g}: \mathrm{X} \rightarrow \mathrm{X}$ are called w-compatible if $\mathrm{g}(\mathrm{F}(\mathrm{x}, \mathrm{y}))=\mathrm{F}(\mathrm{gx}, \mathrm{gy})$ and $\mathrm{g}(\mathrm{F}(\mathrm{y}, \mathrm{x}))=\mathrm{F}(\mathrm{gy}, \mathrm{gx})$, whenever $\mathrm{gx}=\mathrm{F}(\mathrm{x}, \mathrm{y})$ and $\mathrm{gy}=\mathrm{F}(\mathrm{y}, \mathrm{x})$.

Recently, Abbas et al. [8] proved a common fixed point theorem for two maps of Jungck type satisfying generalized condition (B) in metric spaces (See Theorem 2.2, [8]). As a generalization of Theorem 2.2 of [8], Kaewcharoen et al. [1] obtained a common fixed point theorem for four maps satisfying a generalized condition in partial metric spaces. In this paper, we obtain the existence of a unique common coupled fixed point theorem for four mappings satisfying a general contractive condition on partial b-metric-like spaces. We also give an example to illustrate our main theorem. Our theorem generalizes and improves the theorems of [1] and [8].

\section{Main Result}

Theorem 2.1: Let $(\mathrm{X}, \mathrm{p}, \mathrm{k})$ be a complete partial b-metric-like space, $\mathrm{F}, \mathrm{G}: \mathrm{X} \times \mathrm{X} \rightarrow \mathrm{X}$ and $\mathrm{f}, \mathrm{g}: \mathrm{X} \rightarrow \mathrm{X}$ be mappings satisfying

(2.1.1) $\mathrm{F}(\mathrm{X} \times \mathrm{X}) \subseteq \mathrm{g}(\mathrm{X}), \mathrm{G}(\mathrm{X} \times \mathrm{X}) \subseteq \mathrm{f}(\mathrm{X})$,

(2.1.2) $\mathrm{k} \mathrm{p}(\mathrm{F}(\mathrm{x}, \mathrm{y}), \mathrm{G}(\mathrm{u}, \mathrm{v})) \leq \delta M_{u, v}^{x, y}+\mathrm{L} m_{u, v}^{x, y}$

for all $\mathrm{x}, \mathrm{y}, \mathrm{u}, \mathrm{v} \varepsilon \mathrm{X}$, where $\delta>0$ and $\mathrm{L} \geq 0, \mathrm{k} l<1$, where $l=\max \left\{\frac{L}{1-\delta}, \delta+L\right\}$,

(2.1.3) $f(X)$ or $g(X)$ is closed,

(2.1.4) the pairs $(\mathrm{F}, \mathrm{f})$, and $(\mathrm{G}, \mathrm{g})$ are w-compatible.

Then $F, G, f$ and $g$ have a unique common coupled fixed point.

Proof. Let $\left(\mathrm{x}_{0}, \mathrm{y}_{0}\right) \varepsilon \mathrm{X} \times \mathrm{X}$. Since $\mathrm{F}(\mathrm{X} \times \mathrm{X}) \subseteq \mathrm{g}(\mathrm{X})$, there exist $\mathrm{x}_{1}, \mathrm{y}_{1} \varepsilon \mathrm{X}$ such that $\mathrm{gx}_{1}=\mathrm{F}\left(\mathrm{x}_{0}, \mathrm{y}_{0}\right)$ and $\mathrm{gy}_{1}=\mathrm{F}\left(\mathrm{y}_{0}\right.$, $\left.\mathrm{x}_{0}\right)$. Since $\mathrm{G}(\mathrm{X} \times \mathrm{X}) \subseteq \mathrm{f}(\mathrm{X})$, there exist $\mathrm{x}_{2}, \mathrm{y}_{2} \varepsilon X$ such that $\mathrm{fx}_{2}=\mathrm{G}\left(\mathrm{x}_{1}, \mathrm{y}_{1}\right)$ and $\mathrm{fy}_{2}=\mathrm{G}\left(\mathrm{y}_{1}\right.$, $\left.\mathrm{x}_{1}\right)$. Continuing this process, we construct sequences $\left\{\mathrm{x}_{\mathrm{n}}\right\}$ and $\left\{\mathrm{y}_{\mathrm{n}}\right\}$ in $\mathrm{X}$ such that

Now consider

$$
\begin{gathered}
\mathrm{gx}_{2 n+1}=F\left(x_{2 n}, y_{2 n}\right)=z_{2 n}, \\
g_{2 n+1}=F\left(y_{2 n}, x_{2 n}\right)=w_{2 n}, \\
{f x_{2 n+2}}_{=} G\left(x_{2 n+1}, y_{2 n+1}\right)=z_{2 n+1}, \\
f y_{2 n+2}=F\left(y_{2 n+1}, x_{2 n+1}\right)=w_{2 n+1}, n=0,1,2,3, \cdots
\end{gathered}
$$

$\mathrm{p}\left(\mathrm{z}_{2 \mathrm{n}}, \mathrm{z}_{2 \mathrm{n}+1}\right) \leq \mathrm{kp}\left(\mathrm{F}\left(\mathrm{x}_{2 \mathrm{n}}, \mathrm{y}_{2 \mathrm{n}}\right), \mathrm{G}\left(\mathrm{x}_{2 \mathrm{n}+1}, \mathrm{y}_{2 \mathrm{n}+1}\right)\right)$

$$
\leq \delta M_{x_{2 n+1}, y_{2 n+1}}^{x_{2 n}, y_{2 n}}+L \boldsymbol{m}_{x_{2 n+1}, y_{2 n+1}}^{x_{2 n}, y_{2 n}}
$$

where

$$
\begin{gathered}
M_{x_{2 n+1}, y_{2 n+1}}^{x_{2 n}, y_{2 n}}=\max \left\{\begin{array}{c}
\mathrm{p}\left(\mathrm{z}_{2 n-1}, \mathrm{z}_{2 \mathrm{n}}\right), \mathrm{p}\left(\mathrm{w}_{2 n-1}, \mathrm{w}_{2 \mathrm{n}}\right), \mathrm{p}\left(\mathrm{z}_{2 n-1}, \mathrm{z}_{2 \mathrm{n}}\right), \\
\mathrm{p}\left(\mathrm{w}_{2 n-1}, \mathrm{w}_{2 \mathrm{n}}\right), \mathrm{p}\left(\mathrm{z}_{2 \mathrm{n}}, \mathrm{z}_{2 \mathrm{n}+1}\right), \mathrm{p}\left(\mathrm{w}_{2 \mathrm{n}}, \mathrm{w}_{2 \mathrm{n}+1}\right), \\
\frac{1}{2 \mathrm{k}}\left[\mathrm{p}\left(\mathrm{z}_{2 n-1}, \mathrm{z}_{2 \mathrm{n}+1}\right)+\mathrm{p}\left(\mathrm{z}_{2 \mathrm{n}}, \mathrm{z}_{2 \mathrm{n}}\right)\right], \\
\frac{1}{2 \mathrm{k}}\left[\mathrm{p}\left(\mathrm{w}_{2 n-1}, \mathrm{w}_{2 \mathrm{n}+1}\right)+\mathrm{p}\left(\mathrm{w}_{2 \mathrm{n}}, \mathrm{w}_{2 \mathrm{n}}\right)\right]
\end{array}\right\} \\
\leq \max \left\{\begin{array}{c}
\mathrm{p}\left(\mathrm{z}_{2 n-1}, \mathrm{z}_{2 \mathrm{n}}\right), \mathrm{p}\left(\mathrm{w}_{2 n-1}, \mathrm{w}_{2 \mathrm{n}}\right), \\
\mathrm{p}\left(\mathrm{z}_{2 \mathrm{n}}, \mathrm{z}_{2 \mathrm{n}+1}\right), \mathrm{p}\left(\mathrm{w}_{2 \mathrm{n}}, \mathrm{w}_{2 \mathrm{n}+1}\right)
\end{array}\right\} \text { from } \mathrm{k} \geq 1 \text { and from }\left(\mathrm{p}_{4}\right)
\end{gathered}
$$




$$
\begin{aligned}
& m_{x_{2 n+1}, y_{2 n+1}}^{x_{2 n}, y_{2 n}}=\min \left\{\begin{array}{c}
\mathrm{p}\left(\mathrm{z}_{2 n-1}, \mathrm{z}_{2 \mathrm{n}}\right), \mathrm{p}\left(\mathrm{w}_{2 n-1}, \mathrm{w}_{2 \mathrm{n}}\right), \\
\mathrm{p}\left(\mathrm{z}_{2 \mathrm{n}}, \mathrm{z}_{2 \mathrm{n}+1}\right), \mathrm{p}\left(\mathrm{w}_{2 \mathrm{n}}, \mathrm{w}_{2 \mathrm{n}+1}\right), \\
\frac{1}{\mathrm{k}} \mathrm{p}\left(\mathrm{z}_{2 n-1}, \mathrm{z}_{2 \mathrm{n}+1}\right), \frac{1}{\mathrm{k}} \mathrm{p}\left(\mathrm{z}_{2 \mathrm{n}}, \mathrm{z}_{2 \mathrm{n}}\right), \\
\frac{1}{\mathrm{k}} \mathrm{p}\left(\mathrm{w}_{2 n-1}, \mathrm{w}_{2 \mathrm{n}+1}\right), \frac{1}{\mathrm{k}} \mathrm{p}\left(\mathrm{w}_{2 \mathrm{n}}, \mathrm{w}_{2 \mathrm{n}}\right)
\end{array}\right\} \\
& \leq \min \left\{\begin{array}{c}
\mathrm{p}\left(\mathrm{z}_{2 n-1}, \mathrm{z}_{2 \mathrm{n}}\right), \mathrm{p}\left(\mathrm{w}_{2 n-1}, \mathrm{w}_{2 \mathrm{n}}\right), \mathrm{p}\left(\mathrm{z}_{2 \mathrm{n}}, \mathrm{z}_{2 \mathrm{n}+1}\right), \\
\mathrm{p}\left(\mathrm{w}_{2 \mathrm{n}}, \mathrm{w}_{2 \mathrm{n}+1}\right), \mathrm{p}\left(\mathrm{z}_{2 n-1}, \mathrm{z}_{2 \mathrm{n}}\right)+\mathrm{p}\left(\mathrm{z}_{2 n}, \mathrm{z}_{2 \mathrm{n}+1}\right), \\
\mathrm{p}\left(\mathrm{z}_{2 \mathrm{n}}, \mathrm{z}_{2 \mathrm{n}}\right), \mathrm{p}\left(\mathrm{w}_{2 n-1}, \mathrm{w}_{2 \mathrm{n}}\right)+\mathrm{p}\left(\mathrm{w}_{2 n}, \mathrm{w}_{2 \mathrm{n}+1}\right), \mathrm{p}\left(\mathrm{w}_{2 \mathrm{n}}, \mathrm{w}_{2 \mathrm{n}}\right)
\end{array}\right\} \\
& \leq \min \left\{\mathrm{p}\left(\mathrm{z}_{2 \mathrm{n}}, \mathrm{z}_{2 \mathrm{n}}\right), \mathrm{p}\left(\mathrm{w}_{2 \mathrm{n}}, \mathrm{w}_{2 \mathrm{n}}\right)\right\}, \quad \text { from }\left(\mathrm{p}_{2}\right) \\
& \leq \max \left\{\mathrm{p}\left(\mathrm{z}_{2 \mathrm{n}}, \mathrm{z}_{2 \mathrm{n}}\right), \mathrm{p}\left(\mathrm{w}_{2 \mathrm{n}}, \mathrm{w}_{2 \mathrm{n}}\right)\right\} \\
& \leq \max \left\{\mathrm{p}\left(\mathrm{z}_{2 \mathrm{n}}, \mathrm{z}_{2 \mathrm{n}-1}\right), \mathrm{p}\left(\mathrm{w}_{2 \mathrm{n}}, \mathrm{w}_{2 \mathrm{n}-1}\right)\right\}, \quad \text { from }\left(\mathrm{p}_{2}\right) .
\end{aligned}
$$

Thus

Similarly

$$
\mathrm{p}\left(\mathrm{z}_{2 \mathrm{n}}, \mathrm{z}_{2 \mathrm{n}+1}\right) \leq \delta \max \left\{\begin{array}{r}
\mathrm{p}\left(\mathrm{z}_{2 n-1}, \mathrm{z}_{2 \mathrm{n}}\right), \mathrm{p}\left(\mathrm{w}_{2 n-1}, \mathrm{w}_{2 \mathrm{n}}\right), \\
\mathrm{p}\left(\mathrm{z}_{2 \mathrm{n}}, \mathrm{z}_{2 \mathrm{n}+1}\right), \mathrm{p}\left(\mathrm{w}_{2 \mathrm{n}}, \mathrm{w}_{2 \mathrm{n}+1}\right)
\end{array}\right\}+\mathrm{L} \max \left\{\mathrm{p}\left(\mathrm{z}_{2 \mathrm{n}}, \mathrm{z}_{2 \mathrm{n}-1}\right), \mathrm{p}\left(\mathrm{w}_{2 \mathrm{n}}, \mathrm{w}_{2 \mathrm{n}-1}\right)\right\} .
$$

Thus

$$
\mathrm{p}\left(\mathrm{w}_{2 \mathrm{n}}, \mathrm{w}_{2 \mathrm{n}+1}\right) \leq \delta \max \left\{\begin{array}{c}
\mathrm{p}\left(\mathrm{z}_{2 n-1}, \mathrm{z}_{2 \mathrm{n}}\right), \mathrm{p}\left(\mathrm{w}_{2 n-1}, \mathrm{w}_{2 \mathrm{n}}\right), \\
\mathrm{p}\left(\mathrm{z}_{2 \mathrm{n}}, \mathrm{z}_{2 \mathrm{n}+1}\right), \mathrm{p}\left(\mathrm{w}_{2 \mathrm{n}}, \mathrm{w}_{2 \mathrm{n}+1}\right)
\end{array}\right\}+\mathrm{L} \max \left\{\mathrm{p}\left(\mathrm{z}_{2 \mathrm{n}}, \mathrm{z}_{2 \mathrm{n}-1}\right), \mathrm{p}\left(\mathrm{w}_{2 \mathrm{n}}, \mathrm{w}_{2 \mathrm{n}-1}\right)\right\}
$$

$$
\max \left\{\begin{array}{l}
\mathrm{p}\left(\mathrm{z}_{2 n}, \mathrm{z}_{2 \mathrm{n}+1}\right), \\
\mathrm{p}\left(\mathrm{w}_{2 \mathrm{n}}, \mathrm{w}_{2 \mathrm{n}+1}\right)
\end{array}\right\} \leq \delta \max \left\{\begin{array}{c}
\mathrm{p}\left(\mathrm{z}_{2 n-1}, \mathrm{z}_{2 \mathrm{n}}\right), \mathrm{p}\left(\mathrm{w}_{2 n-1}, \mathrm{w}_{2 \mathrm{n}}\right), \\
\mathrm{p}\left(\mathrm{z}_{2 \mathrm{n}}, \mathrm{z}_{2 \mathrm{n}+1}\right), \mathrm{p}\left(\mathrm{w}_{2 \mathrm{n}}, \mathrm{w}_{2 \mathrm{n}+1}\right)
\end{array}\right\}+\mathrm{L} \max \left\{\begin{array}{l}
\mathrm{p}\left(\mathrm{z}_{2 n}, \mathrm{z}_{2 \mathrm{n}-1}\right), \\
\mathrm{p}\left(\mathrm{w}_{2 \mathrm{n}}, \mathrm{w}_{2 \mathrm{n}-1}\right)
\end{array}\right\}
$$

If $\max \left\{\begin{array}{c}\mathrm{p}\left(\mathrm{z}_{2 n-1}, \mathrm{z}_{2 \mathrm{n}}\right), \mathrm{p}\left(\mathrm{w}_{2 n-1}, \mathrm{w}_{2 \mathrm{n}}\right), \\ \mathrm{p}\left(\mathrm{z}_{2 \mathrm{n}}, \mathrm{z}_{2 \mathrm{n}+1}\right), \mathrm{p}\left(\mathrm{w}_{2 \mathrm{n}}, \mathrm{w}_{2 \mathrm{n}+1}\right)\end{array}\right\} \leq \max \left\{\begin{array}{c}\mathrm{p}\left(\mathrm{z}_{2 n}, \mathrm{z}_{2 \mathrm{n}+1}\right), \\ \mathrm{p}\left(\mathrm{w}_{2 \mathrm{n}}, \mathrm{w}_{2 n+1}\right)\end{array}\right\}$,

then from (2)

$$
\max \left\{\mathrm{p}\left(\mathrm{z}_{2 \mathrm{n}}, \mathrm{z}_{2 \mathrm{n}+1}\right), \mathrm{p}\left(\mathrm{w}_{2 \mathrm{n}}, \mathrm{w}_{2 \mathrm{n}+1}\right)\right\} \leq \frac{L}{1-\delta} \max \left\{\mathrm{p}\left(\mathrm{z}_{2 \mathrm{n}-1}, \mathrm{z}_{2 \mathrm{n}}\right), \mathrm{p}\left(\mathrm{w}_{2 \mathrm{n}-1}, \mathrm{w}_{2 \mathrm{n}}\right)\right\} .
$$

If $\quad \max \left\{\begin{array}{r}\mathrm{p}\left(\mathrm{z}_{2 n-1}, \mathrm{z}_{2 \mathrm{n}}\right), \mathrm{p}\left(\mathrm{w}_{2 n-1}, \mathrm{w}_{2 \mathrm{n}}\right), \\ \mathrm{p}\left(\mathrm{z}_{2 \mathrm{n}}, \mathrm{z}_{2 \mathrm{n}+1}\right), \mathrm{p}\left(\mathrm{w}_{2 \mathrm{n}}, \mathrm{w}_{2 \mathrm{n}+1}\right)\end{array}\right\} \leq \max \left\{\begin{array}{c}\mathrm{p}\left(\mathrm{z}_{2 n-1}, \mathrm{z}_{2 \mathrm{n}}\right), \\ \mathrm{p}\left(\mathrm{w}_{2 \mathrm{n}-1}, \mathrm{w}_{2 \mathrm{n}}\right)\end{array}\right\}$,

then from (2)

Hence

$$
\max \left\{\mathrm{p}\left(\mathrm{z}_{2 \mathrm{n}}, \mathrm{z}_{2 \mathrm{n}+1}\right), \mathrm{p}\left(\mathrm{w}_{2 \mathrm{n}}, \mathrm{w}_{2 \mathrm{n}+1}\right)\right\} \leq(\delta+\mathrm{L}) \max \left\{\mathrm{p}\left(\mathrm{z}_{2 \mathrm{n}-1}, \mathrm{z}_{2 \mathrm{n}}\right), \mathrm{p}\left(\mathrm{w}_{2 \mathrm{n}-1}, \mathrm{w}_{2 \mathrm{n}}\right)\right\} .
$$

where $l=\max \left\{\frac{L}{1-\delta}, \delta+L\right\}<1$.

Similarly we can show that

Hence

$\max \left\{\mathrm{p}\left(\mathrm{z}_{2 \mathrm{n}-1}, \mathrm{z}_{2 \mathrm{n}}\right), \mathrm{p}\left(\mathrm{w}_{2 \mathrm{n}-1}, \mathrm{w}_{2 \mathrm{n}}\right)\right\} \leq l \max \left\{\mathrm{p}\left(\mathrm{z}_{2 \mathrm{n}-2}, \mathrm{z}_{2 \mathrm{n}-1}\right), \mathrm{p}\left(\mathrm{w}_{2 \mathrm{n}-2}, \mathrm{w}_{2 \mathrm{n}-1}\right)\right\}$.

Thus

From (3), it follows that

$$
\operatorname{Max}\left\{\mathrm{p}\left(\mathrm{z}_{\mathrm{n}}, \mathrm{z}_{\mathrm{n}+1}\right), \mathrm{p}\left(\mathrm{w}_{\mathrm{n}}, \mathrm{w}_{\mathrm{n}+1}\right)\right\} \leq l \max \left\{\mathrm{p}\left(\mathrm{z}_{\mathrm{n}-1}, \mathrm{z}_{\mathrm{n}}\right), \mathrm{p}\left(\mathrm{w}_{\mathrm{n}-1}, \mathrm{w}_{\mathrm{n}}\right)\right\}, \mathrm{n}=1,2,3, \cdots
$$

$$
\max \left\{\mathrm{p}\left(\mathrm{z}_{\mathrm{n}}, \mathrm{z}_{\mathrm{n}+1}\right), \mathrm{p}\left(\mathrm{w}_{\mathrm{n}}, \mathrm{w}_{\mathrm{n}+1}\right)\right\} \leq l^{\mathrm{n}} \max \left\{\mathrm{p}\left(\mathrm{z}_{0}, \mathrm{z}_{1}\right), \mathrm{p}\left(\mathrm{w}_{0}, \mathrm{w}_{1}\right)\right\} .
$$

$$
\lim _{n \rightarrow \infty} \mathrm{p}\left(\mathrm{z}_{\mathrm{n}}, \mathrm{z}_{\mathrm{n}+1}\right)=0=\lim _{n \rightarrow \infty} \mathrm{p}\left(\mathrm{w}_{\mathrm{n}}, \mathrm{w}_{\mathrm{n}+1}\right)
$$

For $\mathrm{m}>\mathrm{n}$, consider

$\max \left\{\mathrm{p}\left(\mathrm{z}_{\mathrm{n}}, \mathrm{z}_{\mathrm{m}}\right), \mathrm{p}\left(\mathrm{w}_{\mathrm{n}}, \mathrm{w}_{\mathrm{m}}\right)\right\}$

$$
\leq \max \left\{\begin{array}{c}
\mathrm{k} \mathrm{p}\left(\mathrm{z}_{\mathrm{n}}, \mathrm{z}_{\mathrm{n}+1}\right)+\mathrm{k}^{2} \mathrm{p}\left(\mathrm{z}_{\mathrm{n}+1}, \mathrm{z}_{\mathrm{n}+2}\right)+\ldots+\mathrm{k}^{\mathrm{m}-\mathrm{n}-1} \mathrm{p}\left(\mathrm{z}_{\mathrm{m}-2}, \mathrm{z}_{\mathrm{m}-1}\right)+\mathrm{k}^{\mathrm{m}-\mathrm{n}-1} \mathrm{p}\left(\mathrm{z}_{\mathrm{m}-1}, \mathrm{z}_{\mathrm{m}}\right), \\
\mathrm{kp}\left(\mathrm{w}_{\mathrm{n}}, \mathrm{w}_{\mathrm{n}+1}\right)+\mathrm{k}^{2} \mathrm{p}\left(\mathrm{w}_{\mathrm{n}+1}, \mathrm{w}_{\mathrm{n}+2}\right)+\ldots+\mathrm{k}^{\mathrm{m}-\mathrm{n}-1} \mathrm{p}\left(\mathrm{w}_{\mathrm{m}-2}, \mathrm{w}_{\mathrm{m}-1}\right)+\mathrm{k}^{\mathrm{m}-\mathrm{n}-1} \mathrm{p}\left(\mathrm{w}_{\mathrm{m}-1}, \mathrm{w}_{\mathrm{m}}\right)
\end{array}\right\}
$$

$\leq \mathrm{k} \max \left\{\mathrm{p}\left(\mathrm{z}_{\mathrm{n}}, \mathrm{z}_{\mathrm{n}+1}\right), \mathrm{p}\left(\mathrm{w}_{\mathrm{n}}, \mathrm{w}_{\mathrm{n}+1}\right)\right\}+\mathrm{k}^{2} \max \left\{\mathrm{p}\left(\mathrm{z}_{\mathrm{n}+1}, \mathrm{z}_{\mathrm{n}+2}\right), \mathrm{p}\left(\mathrm{w}_{\mathrm{n}+1}, \mathrm{w}_{\mathrm{n}+2}\right)\right\}$

$$
\begin{aligned}
& +\ldots+\mathrm{k}^{\mathrm{m}-\mathrm{n}-1} \max \left\{\mathrm{p}\left(\mathrm{z}_{\mathrm{m}-2}, \mathrm{z}_{\mathrm{m}-1}\right), \mathrm{p}\left(\mathrm{w}_{\mathrm{m}-2}, \mathrm{w}_{\mathrm{m}-1}\right)\right\}+ \\
& \mathrm{k}^{\mathrm{m}-\mathrm{n}-1} \max \left\{\mathrm{p}\left(\mathrm{z}_{\mathrm{m}-1}, \mathrm{z}_{\mathrm{m}}\right), \mathrm{p}\left(\mathrm{w}_{\mathrm{m}-1}, \mathrm{w}_{\mathrm{m}}\right)\right\}
\end{aligned}
$$




$$
\begin{aligned}
& \leq\left(k l^{n}+k^{2} l^{n+1}+\ldots .+k^{m-n-1} l^{m-2}+k^{m-n-1} l^{m-1}\right) \max \left\{\begin{array}{l}
\mathrm{p}\left(\mathrm{z}_{0}, \mathrm{z}_{1}\right), \\
\mathrm{p}\left(\mathrm{w}_{0}, \mathrm{w}_{1}\right)
\end{array}\right\} \\
& =k l^{n}\left(1+k l^{1}+k^{2} l^{2}+\ldots .+k^{m-n-2} l^{m-n-2}+k^{m-n-2} l^{m-n-1}\right) \max \left\{\begin{array}{l}
\mathrm{p}\left(\mathrm{z}_{0}, \mathrm{z}_{1}\right), \\
\mathrm{p}\left(\mathrm{w}_{0}, \mathrm{w}_{1}\right)
\end{array}\right\} \leq \\
& k l^{n}\left(1+k l^{1}+k^{2} l^{2}+\ldots .+k^{m-n-2} l^{m-n-2}+k^{m-n-1} l^{m-n-1}\right) \max \left\{\begin{array}{l}
\mathrm{p}\left(\mathrm{z}_{0}, \mathrm{z}_{1}\right), \\
\mathrm{p}\left(\mathrm{w}_{0}, \mathrm{w}_{1}\right)
\end{array}\right\} \\
& \leq \frac{k l^{n}}{1-k l} \max \left\{\begin{array}{l}
\mathrm{p}\left(\mathrm{z}_{0}, \mathrm{z}_{1}\right), \\
\mathrm{p}\left(\mathrm{w}_{0}, \mathrm{w}_{1}\right)
\end{array}\right\}, \text { since } \mathrm{k} l<1 .
\end{aligned}
$$

Hence

$$
\lim _{n, m \rightarrow \infty} \mathrm{p}\left(\mathrm{z}_{\mathrm{n}}, \mathrm{z}_{\mathrm{m}}\right)=0=\lim _{n, m \rightarrow \infty} \mathrm{p}\left(\mathrm{w}_{\mathrm{n}}, \mathrm{w}_{\mathrm{m}}\right) .
$$

Thus $\left\{z_{n}\right\}$ and $\left\{w_{n}\right\}$ are Cauchy in $(X, p, k)$.

Since $X$ is complete, the sequences $\left\{z_{n}\right\}$ and $\left\{w_{n}\right\}$ converge to some $\alpha$ and $\beta$ in $X$ respectively such that

$\lim _{n, m \rightarrow \infty} \mathrm{p}\left(\mathrm{z}_{\mathrm{n}}, \mathrm{z}_{\mathrm{m}}\right)=\mathrm{p}(\alpha, \alpha)$ and $\lim _{n, m \rightarrow \infty} \mathrm{p}\left(\mathrm{w}_{\mathrm{n}}, \mathrm{w}_{\mathrm{m}}\right)=\mathrm{p}(\beta, \beta)$.

Also $\lim _{n \rightarrow \infty} \mathrm{p}\left(\mathrm{z}_{\mathrm{n}}, \alpha\right)=\mathrm{p}(\alpha, \alpha)$ and $\lim _{n \rightarrow \infty} \mathrm{p}\left(\mathrm{w}_{\mathrm{n}}, \beta\right)=\mathrm{p}(\beta, \beta)$.

Now from (5), we have

Suppose $\mathrm{f}(\mathrm{X})$ is closed.

$$
\mathrm{p}(\alpha, \alpha)=0=\mathrm{p}(\beta, \beta)
$$

Since $\mathrm{fx}_{2 \mathrm{n}+2}=\mathrm{z}_{2 \mathrm{n}+1} \rightarrow \alpha$ and $\mathrm{fy}_{2 \mathrm{n}+2}=\mathrm{w}_{2 \mathrm{n}+1} \rightarrow \beta$, it follows that $\alpha=\mathrm{fu}$ and $\beta=$ fv for some $u, v \varepsilon X$.

Consider

$$
\begin{aligned}
& \begin{array}{l}
\mathrm{p}(\alpha, \mathrm{F}(\mathrm{u}, \mathrm{v})) \leq \mathrm{kp}\left(\alpha, \mathrm{z}_{2 \mathrm{n}+1}\right)+\mathrm{kp}\left(\mathrm{F}(\mathrm{u}, \mathrm{v}), \mathrm{G}\left(\mathrm{x}_{2 \mathrm{n}+1}, \mathrm{y}_{2 \mathrm{n}+1}\right)\right) \\
\leq \mathrm{kp}\left(\alpha, \mathrm{z}_{2 \mathrm{n}+1}\right)+\delta M_{x_{2 n+1}, y_{2 n+1}}^{u, \mathrm{~L} m_{x_{2 n+1}, y_{2 n+1}}^{u, v}}
\end{array} \\
& \left.M_{x_{2 n+1}, y_{2 n+1}}^{u, v}=\max \left\{\begin{array}{c}
\mathrm{p}\left(\mathrm{fu}, \mathrm{z}_{2 \mathrm{n}}\right), \mathrm{p}\left(\mathrm{fv}, \mathrm{w}_{2 \mathrm{n}}\right), \mathrm{p}(\mathrm{fu}, \mathrm{F}(\mathrm{u}, \mathrm{v})), \\
\mathrm{p}(\mathrm{fv}, \mathrm{F}(\mathrm{v}, \mathrm{u})), \mathrm{p}\left(\mathrm{z}_{2 \mathrm{n}}, \mathrm{z}_{2 \mathrm{n}+1}\right), \mathrm{p}\left(\mathrm{w}_{2 \mathrm{n}}, \mathrm{w}_{2 \mathrm{n}+1}\right), \\
\frac{1}{2 \mathrm{k}}\left[\mathrm{p}\left(\mathrm{fu}, \mathrm{z}_{2 \mathrm{n}+1}\right)+\mathrm{p}\left(\mathrm{z}_{2 \mathrm{n}}, \mathrm{F}(\mathrm{u}, \mathrm{v})\right)\right], \\
\frac{1}{2 \mathrm{k}}\left[\mathrm{p}\left(\mathrm{fv}, \mathrm{w}_{2 \mathrm{n}+1}\right)+\mathrm{p}\left(\mathrm{w}_{2 \mathrm{n}}, \mathrm{F}(\mathrm{v}, \mathrm{u})\right]\right.
\end{array}\right\}\right) \\
& \left.\rightarrow \max \left\{\begin{array}{c}
0,0, \mathrm{p}(\alpha, \mathrm{F}(\mathrm{u}, \mathrm{v})), \\
\mathrm{p}(\beta, \mathrm{F}(\mathrm{v}, \mathrm{u})), 0,0, \\
\frac{1}{2 \mathrm{k}}[0+\mathrm{p}(\alpha, \mathrm{F}(\mathrm{u}, \mathrm{v}))], \\
\frac{1}{2 \mathrm{k}}[0+\mathrm{p}(\beta, \mathrm{F}(\mathrm{v}, \mathrm{u})]
\end{array}\right\}, \text { from }(4) \text { and Remark } 1.7 \text { (ii) }\right) \\
& \text { Also } m_{x_{2 n+1}, y_{2 n+1}}^{\rightarrow, v} 0 .
\end{aligned}
$$

Thus

Similarly we can show that

$$
\mathrm{p}(\alpha, \mathrm{F}(\mathrm{u}, \mathrm{v})) \leq \delta \max \{\mathrm{p}(\alpha, \mathrm{F}(\mathrm{u}, \mathrm{v})), \mathrm{p}(\beta, \mathrm{F}(\mathrm{v}, \mathrm{u}))\}
$$

Hence

$$
\mathrm{p}(\beta, \mathrm{F}(\mathrm{u}, \mathrm{v})) \leq \delta \max \{\mathrm{p}(\alpha, \mathrm{F}(\mathrm{u}, \mathrm{v})), \mathrm{p}(\beta, \mathrm{F}(\mathrm{v}, \mathrm{u}))\}
$$

$\max \{\mathrm{p}(\alpha, \mathrm{F}(\mathrm{u}, \mathrm{v})), \mathrm{p}(\beta, \mathrm{F}(\mathrm{v}, \mathrm{u}))\} \leq \delta \max \{\mathrm{p}(\alpha, \mathrm{F}(\mathrm{u}, \mathrm{v})), \mathrm{p}(\beta, \mathrm{F}(\mathrm{v}, \mathrm{u}))\}$,

which in turn yields that $\alpha=\mathrm{F}(\mathrm{u}, \mathrm{v})$ and $\beta=\mathrm{F}(\mathrm{v}, \mathrm{u})$.

Thus $\mathrm{fu}=\alpha=\mathrm{F}(\mathrm{u}, \mathrm{v})$ and $\mathrm{fv}=\beta=\mathrm{F}(\mathrm{v}, \mathrm{u})$.

Since the pair $(\mathrm{F}, \mathrm{f})$ is $\mathrm{w}$ - compatible, we have

$$
f \alpha=F(\alpha, \beta) \text { and } f \beta=F(\beta, \alpha) .
$$

Since $\alpha=\mathrm{F}(\mathrm{u}, \mathrm{v}) \varepsilon \mathrm{F}(\mathrm{X} \times \mathrm{X}) \subseteq \mathrm{g}(\mathrm{X})$, there exists $\mathrm{r} \varepsilon \mathrm{X}$ such that $\alpha=$ gr.

Since $\beta=\mathrm{F}(\mathrm{v}, \mathrm{u}) \varepsilon \mathrm{F}(\mathrm{X} \times \mathrm{X}) \subseteq \mathrm{g}(\mathrm{X})$, there exists $\mathrm{t} \varepsilon \mathrm{X}$ such that $\beta=\mathrm{gt}$. 
Now $\mathrm{p}(\alpha, \mathrm{G}(\mathrm{r}, \mathrm{t})) \leq \mathrm{s} \mathrm{p}(\mathrm{F}(\mathrm{u}, \mathrm{v}), \mathrm{G}(\mathrm{r}, \mathrm{t})) \leq \delta \boldsymbol{M}_{r, t}^{u, v}+\mathrm{L} \boldsymbol{m}_{r, t}^{u, v}$

$$
\begin{gathered}
M_{r, t}^{u, v}=\max \left\{\begin{array}{r}
\mathrm{p}(\mathrm{fu}, \mathrm{gr}), \mathrm{p}(\mathrm{fv}, \mathrm{gt}), \mathrm{p}(\mathrm{fu}, \mathrm{F}(\mathrm{u}, \mathrm{v})), \\
\mathrm{p}(\mathrm{fv}, \mathrm{F}(\mathrm{v}, \mathrm{u})), \mathrm{p}(\mathrm{gr}, \mathrm{G}(\mathrm{r}, \mathrm{t})), \mathrm{p}(\mathrm{gt}, \mathrm{G}(\mathrm{t}, \mathrm{r})), \\
\frac{1}{2 \mathrm{k}}[\mathrm{p}(\mathrm{fu}, \mathrm{G}(\mathrm{r}, \mathrm{t}))+\mathrm{p}(\mathrm{gr}, \mathrm{F}(\mathrm{u}, \mathrm{v}))], \\
\frac{1}{2 \mathrm{k}}[\mathrm{p}(\mathrm{fv}, \mathrm{G}(\mathrm{t}, \mathrm{r}))+\mathrm{p}(\mathrm{gt}, \mathrm{F}(\mathrm{v}, \mathrm{u})] \\
0,0,0,0)
\end{array}\right\} \\
=\max \left\{\begin{array}{c}
\mathrm{p}(\alpha, \mathrm{G}(\mathrm{r}, \mathrm{t})), \mathrm{p}(\beta, \mathrm{G}(\mathrm{t}, \mathrm{r})), \\
\frac{1}{2 \mathrm{k}}[\mathrm{p}(\alpha, \mathrm{G}(\mathrm{r}, \mathrm{t}))+\mathrm{p}(\beta, \mathrm{F}(\mathrm{u}, \mathrm{v}))], \\
\frac{1}{2 \mathrm{k}}[\mathrm{p}(\beta, \mathrm{G}(\mathrm{t}, \mathrm{r}))+\mathrm{p}(\beta, \mathrm{F}(\mathrm{v}, \mathrm{u})]
\end{array}\right\}
\end{gathered}
$$

$m_{r, t}^{u, v}=0$.

Thus

Similarly we can show that

$$
\mathrm{p}(\alpha, \mathrm{G}(\mathrm{r}, \mathrm{t})) \leq \delta \max \{\mathrm{p}(\alpha, \mathrm{G}(\mathrm{r}, \mathrm{t})), \mathrm{p}(\beta, \mathrm{G}(\mathrm{t}, \mathrm{r}))\}
$$$$
\mathrm{p}(\beta, \mathrm{G}(\mathrm{t}, \mathrm{r})) \leq \delta \max \{\mathrm{p}(\alpha, \mathrm{G}(\mathrm{r}, \mathrm{t})), \mathrm{p}(\beta, \mathrm{G}(\mathrm{t}, \mathrm{r}))\} .
$$

Hence

$$
\max \{\mathrm{p}(\alpha, \mathrm{G}(\mathrm{r}, \mathrm{t})), \mathrm{p}(\beta, \mathrm{G}(\mathrm{t}, \mathrm{r}))\} \leq \delta \max \{\mathrm{p}(\alpha, \mathrm{G}(\mathrm{r}, \mathrm{t})), \mathrm{p}(\beta, \mathrm{G}(\mathrm{t}, \mathrm{r}))\}
$$

which in turn yields that $\mathrm{gr}=\alpha=\mathrm{G}(\mathrm{r}, \mathrm{t})$ and $\mathrm{gt}=\beta=\mathrm{G}(\mathrm{t}, \mathrm{r})$.

Since the pair $(\mathrm{G}, \mathrm{g})$ is w-compatible, we have

Now consider

$$
\mathrm{g} \alpha=\mathrm{G}(\alpha, \beta) \text { and } \mathrm{g} \beta=\mathrm{G}(\beta, \alpha) .
$$

$$
\begin{gathered}
\mathrm{p}(\mathrm{f} \alpha, \alpha) \leq \mathrm{kp}(\mathrm{F}(\alpha, \beta), \mathrm{G}(\mathrm{r}, \mathrm{t})) \leq \delta M_{r, t}^{\alpha, \beta}+\mathrm{L} \boldsymbol{m}_{r, t}^{\alpha, \beta} \\
M_{r, t}^{\alpha, \beta}=\max \left\{\begin{array}{c}
\mathrm{p}(\mathrm{f} \alpha, \mathrm{gr}), \mathrm{p}(\mathrm{f} \beta, \mathrm{gt}), \mathrm{p}(\mathrm{f} \alpha, \mathrm{F}(\alpha, \beta)), \\
\mathrm{p}(\mathrm{f} \beta, \mathrm{F}(\beta, \alpha)), \mathrm{p}(\mathrm{gr}, \mathrm{G}(\mathrm{r}, \mathrm{t})), \mathrm{p}(\mathrm{gt}, \mathrm{G}(\mathrm{t}, \mathrm{r})), \\
\frac{1}{2 \mathrm{k}}[\mathrm{p}(\mathrm{f} \alpha, \mathrm{G}(\mathrm{r}, \mathrm{t}))+\mathrm{p}(\mathrm{gr}, \mathrm{F}(\alpha, \beta))], \\
\frac{1}{2 \mathrm{k}}[\mathrm{p}(\mathrm{f} \beta, \mathrm{G}(\mathrm{t}, \mathrm{r}))+\mathrm{p}(\mathrm{gt}, \mathrm{F}(\beta, \alpha))]
\end{array}\right\} \\
=\max \left\{\begin{array}{c}
\frac{1}{2 \mathrm{k}}[\mathrm{p}(\mathrm{f} \alpha, \alpha)+\mathrm{p}(\alpha, \mathrm{f} \alpha)], \\
\mathrm{p}(\mathrm{f} \alpha, \alpha), \mathrm{p}(\mathrm{f} \beta, \beta), 0,0,0,0, \\
\frac{1}{2 \mathrm{k}}[\mathrm{p}(\mathrm{f} \beta, \beta)+\mathrm{p}(\beta, \mathrm{f} \beta)]
\end{array}\right\} \\
=\max \{\mathrm{p}(\mathrm{f} \alpha, \alpha), \mathrm{p}(\mathrm{f} \beta, \beta)\} .
\end{gathered}
$$

Also $\boldsymbol{m}_{r, t}^{\alpha, \beta}=0$.

Thus $\mathrm{p}(\mathrm{f} \alpha, \alpha) \leq \delta \max \{\mathrm{p}(\mathrm{f} \alpha, \alpha), \mathrm{p}(\mathrm{f} \beta, \beta)\}$.

Similarly we can show that

Hence

$\mathrm{p}(\mathrm{f} \beta, \beta) \leq \delta \max \{\mathrm{p}(\mathrm{f} \alpha, \alpha), \mathrm{p}(\mathrm{f} \beta, \beta)\}$.

$\max \{\mathrm{p}(\mathrm{f} \alpha, \alpha), \mathrm{p}(\mathrm{f} \beta, \beta)\} \leq \delta \max \{\mathrm{p}(\mathrm{f} \alpha, \alpha), \mathrm{p}(\mathrm{f} \beta, \beta)\}$

which in turn yields that $f \alpha=\alpha$ and $\mathrm{f} \beta=\beta$.

Similarly we can show that $\mathrm{g} \alpha=\alpha$ and $\mathrm{g} \beta=\beta$.

Thus 


\section{A UNIQUE COMMON COUPLED FIXED POINT THEOREM}

$F(\alpha, \beta)=f \alpha=\alpha=g \alpha=G(\alpha, \beta)$ and

$\mathrm{F}(\beta, \alpha)=\mathrm{f} \beta=\beta=\mathrm{g} \beta=\mathrm{G}(\beta, \alpha)$.

Hence $(\alpha, \beta)$ is a common coupled fixed point of $\mathrm{F}, \mathrm{G}, \mathrm{f}$ and $\mathrm{g}$. Uniqueness of this common coupled fixed point follows easily from (2.1.2).

Now, we give an example to illustrate our main Theorem 2.1 .

Example 2.2 Let $X=[0,1]$ and $p(x, y)=\max \left\{x^{2}, y^{2}\right\}$. Then $(X, p, k)$ is a complete partial b-metric-like space with $\mathrm{k}=2$. Define $\mathrm{F}, \mathrm{G}: \mathrm{X} \times \mathrm{X} \rightarrow \mathrm{X}$ and $\mathrm{f}, \mathrm{g}: \mathrm{X} \rightarrow \mathrm{X}$ as $\mathrm{F}(\mathrm{x}, \mathrm{y})=0, \mathrm{G}(\mathrm{x}, \mathrm{y})=\frac{x}{4}$, $\mathrm{fx}=\frac{x}{2}$ and $\mathrm{gx}=\mathrm{x}$. Then

$\mathrm{kp}(\mathrm{F}(\mathrm{x}, \mathrm{y}), \mathrm{G}(\mathrm{u}, \mathrm{v}))=2 \max \left\{0, \frac{u^{2}}{16}\right\}=\frac{u^{2}}{8}$,

$\mathrm{p}(\mathrm{gu}, \mathrm{G}(\mathrm{u}, \mathrm{v}))=\max \left\{u^{2}, \frac{u^{2}}{16}\right\}=\mathrm{u}^{2}$.

Thus $\mathrm{p}(\mathrm{F}(\mathrm{x}, \mathrm{y}), \mathrm{G}(\mathrm{u}, \mathrm{v}))=\frac{1}{8} \mathrm{u}^{2}=\frac{1}{8} \mathrm{p}(\mathrm{gu}, \mathrm{G}(\mathrm{u}, \mathrm{v}))$

$$
\leq \frac{1}{8} M_{u, v}^{x, y}+0 m_{u, v}^{x, y}
$$

Here $\delta=\frac{1}{8}, \mathrm{~L}=0, \mathrm{k} l=\frac{1}{4}<1$. Clearly (2.1.1), (2.1.3) and (2.1.4) are satisfied and (0,0) is the unique common coupled fixed point of $\mathrm{F}, \mathrm{G}, \mathrm{f}$ and $\mathrm{g}$.

Theorem 2.1 is a generalization and improvement of the following:

Theorem 2.3 (Theorem 2.1, [1]): Let $(X, p)$ be a complete partial metric space. Suppose that $f, g, F, G: X \rightarrow X$ satisfying the following conditions

(2.3.1) $\mathrm{f}(\mathrm{X}) \subseteq \mathrm{g}(\mathrm{X})$ and $\mathrm{F}(\mathrm{X}) \subseteq \mathrm{G}(\mathrm{X})$,

(2.3.2) there exist $\delta>0$ and $\mathrm{L} \geq 0$ with $\delta+2 \mathrm{~L}<1$ such that

$\mathrm{p}(\mathrm{Fx}, \mathrm{fy}) \leq \delta \mathrm{M}(\mathrm{x}, \mathrm{y})+\mathrm{L} \min \{\mathrm{p}(\mathrm{gx}, \mathrm{Fx}), \mathrm{p}(\mathrm{Gy}, \mathrm{fy}), \mathrm{p}(\mathrm{gx}, \mathrm{fy}), \mathrm{Gy}, \mathrm{Fx})\}$

for all $\mathrm{x}, \mathrm{y} \varepsilon \mathrm{X}$, where

$M(x, y)=\max \left\{p(g x, G y), p(g x, F x), p(G y, f y), \frac{1}{2}[p(g x, f y)+p(G y, F x)]\right\}$,

(2.3.3) $\mathrm{f}(\mathrm{X})$ or $\mathrm{g}(\mathrm{X})$ is closed and

(2.3.4) the pairs (f, G) and (g, F ) are w-compatible.

Then $\mathrm{f}, \mathrm{g}, \mathrm{F}$ and $\mathrm{G}$ have a unique common fixed point in $\mathrm{X}$.

\section{Acknowledgement}

The authors are thankful to the referees for their valuable suggestions in improving the manuscript.

\section{References}

1. Kaewcharoen, A. and Yuying, T. Unique common fixed point theorems on partial metric spaces. J. Nonlinear Sciences and Applications, 2014, 7, 90-101.

2. Czerwik, S. Contraction mappings in b-metric spaces. Acta Math. Inf. Univ. Ostraviensis, 1993, 1, 5-11.

3. Alghamdi, M., Ali Hussain, N. and Salimi, P. Fixed point and coupled fixed point theorems on b-metric-like spaces. J. Inequalities and Applications, 2013, 402, 25 pages.

4. Matthews, S.G. Partial metric topology, Proc. 8th Summer conference on General Topology and Applications. Ann. New York Acad. Sci., 1994, 728, 183-197.

5. Bhaskar, T.G. and Lakshmikantham, V. Fixed point theorems in partially ordered metric spaces and applications. Nonlinear Anal., 2006, 65(7), 1379-1393.

6. Lakshmikantham, V. and Ciric, Lj. Coupled fixed point theorems for non-linear contractions in partially ordered metric spaces, Nonlinear Analysis: Theory, Methods and Applications, 2009, 70(12), 4341-4349.

7. Abbas, M.A.. Khan, M. and Radenovic, S. Common coupled fixed point theorems in cone metric spaces for w-compatible mappings. Appl. Mathematics and Computation, 2010, 217(1), 195-202.

8. Abbas, M. Babu, G.V.R. and Alemayelu, G.N. On common fixed points of weakly compatible mappings satisfying generalized condition(B). Filomat, 2011, 25(2), 9-19. 